\title{
Extended Abstract: Deficiency of Sodium Taurocholate Cotransporting Polypeptide (SLC10A1): A New Inborn Error of Metabolism with an Attenuated Phenotype
}

\author{
Frédéric M. Vaz ${ }^{a}$ Hidde H. Huidekoper ${ }^{c}$ Coen C. Paulusma ${ }^{b}$

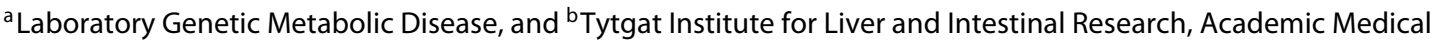 \\ Center, Amsterdam, and ' Department of Pediatrics, Center for Lysosomal and Metabolic Diseases, Erasmus Medical \\ Center, Rotterdam, The Netherlands
}

\section{Keywords}

$\mathrm{Na}$-taurocholate cotransporting polypeptide $\cdot$ Bile salts .

Enterohepatic circulation - Inborn error of metabolism

\begin{abstract}
We present the first patient with a defect in the $\mathrm{Na}^{+}$-taurocholate cotransporting polypeptide SLC10A1 (NTCP), which plays a key role in the enterohepatic circulation of bile salts. The clinical presentation of the child was mild and the child showed no signs of liver dysfunction or pruritus despite extremely elevated plasma bile salt levels (>100-fold upperlimit of normal). A homozygous point mutation was found in the SLC10A1 gene (resulting in amino acid change R252H) and functional studies confirmed the pathogenicity of the mutation. This confirms the role of NTCP as the major transporter of conjugated bile salts into the liver as part of the enterohepatic circulation and shows that other transporters partly can take over its function, resulting in a relatively mild phenotype. This work was published previously in [Vaz et al.: Hepatology 2015;61:260-267] and supplemented with some follow-up information of the patient.
\end{abstract}

(c) 2017 S. Karger AG, Basel
(C) 2017 S. Karger AG, Basel

KARGER

E-Mail karger@karger.com

www.karger.com/ddi

\section{Introduction}

The enterohepatic circulation of bile salts is an important physiological route to recycle bile salts and ensure intestinal absorption of dietary lipids. The $\mathrm{Na}^{+}$-taurocholate cotransporting polypeptide SLC10A1 (NTCP) plays a key role in this process as the major transporter of conjugated bile salts from the plasma compartment into the hepatocyte. Several disorders associated with defects in the enterohepatic circulation of bile salts have been described (e.g. PFIC 1, 2 and 3), which all lead to severe liver dysfunction. Here, we present the first patient with NTCP deficiency with a surprisingly mild phenotype.

\section{Patient Description and Diagnosis}

A girl of 2 years presented with mild hypotonia, growth retardation and delayed motor milestones. Total bile salts in plasma were extremely elevated (up to $1,500 \mu \mathrm{M}$, ref. $<16.3$ ), but there were no clinical signs of cholestatic jaundice, pruritus or liver dysfunction. Bile salt synthesis and intestinal bile salt signaling were not affected as evidenced 
Fig. 1. Time profile of cholic acid (CA), chenodeoxycholic acid (CDCA) and total bile salt levels from 2009 to 2016 in the NTCP-deficient patient. The age of the patient in years is indicated (blue arrow) as well as the reference upper-limit of normal (Ref. ULN) of total bile salt levels (red dashed line).

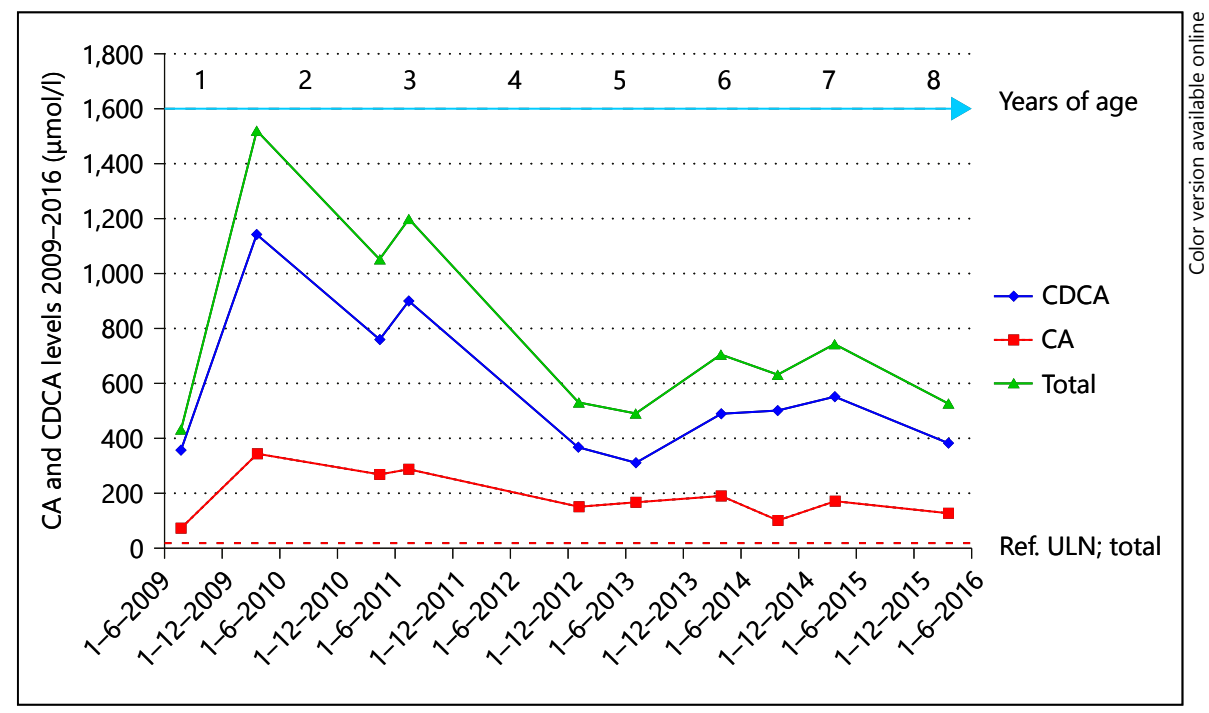

by normal plasma 7a-hydroxy-4-cholesten-3-one (C4) and FGF19 levels, respectively. The presence of secondary bile salts in the circulation suggested residual enterohepatic circulation of bile salts. Sequencing of the SLC1OA 1 gene revealed a single homozygous, non-synonymous point mutation in the coding sequence of the gene resulting in an arginine to histidine substitution at position 252. In vitro studies showed that the mutant protein was virtually absent from the plasma membrane with concomitant reduced uptake of taurocholic acid. Surface expression of the mutant protein and consequent transport activity could be partially restored by the proteostasis regulator SAHA.

\section{Three-Year Follow-Up}

The child is developmentally delayed, but this is not progressive and she attends special education. There still are no signs of pruritus or steatorrhea and both height and weight develop normally, which is no indication of malabsorption leading to growth retardation. Alanine aminotransferase has been increasing from normal to mildly elevated (2-times upper-limit of normal), and aspartate aminotransferase and total bilirubin levels are sometimes (mildly) elevated. The prothrombin time is consistently mildly elevated $(\sim 13.5, \mathrm{n}<11.9 \mathrm{~s})$ suggesting vitamin $\mathrm{K}$ deficiency to a certain extent. Other fat-soluble vitamins are present at normal levels or are slightly lowered. Total bile salt levels remain grossly elevated, but there appears to be a trend towards lower values (fig. 1). In the first 3-4 years of life, the total bile salt levels were well over 1,000 $\mu \mathrm{mol} / \mathrm{l}$, but after her fifth birthday, levels have declined and varied between 500 and $800 \mu \mathrm{mol} / \mathrm{l}$ $(\mathrm{n}<20 \mu \mathrm{mol} / \mathrm{l})$.

\section{Conclusion}

In conclusion, we describe the identification of NTCP deficiency as a new inborn error of metabolism with a relatively mild clinical phenotype that during follow-up has not progressed and remains attenuated. The identification of NTCP deficiency confirms that this transporter is the main import system for conjugated bile salts into the liver but also suggests that auxiliary transporters are able to sustain the enterohepatic circulation in its absence.

\section{Disclosure Statement}

The authors declare no conflict of interest.

Reference

1 Vaz FM, Paulusma CC, Huidekoper H, de Ru M, Lim C, Koster J, et al: Sodium taurocholate cotransporting polypeptide (SLC10A1) deficiency: conjugated hypercholanemia without a clear clinical phenotype. Hepatology 2015; 61:260-267. 